\title{
Pericardial effusion caused by accidently placing a MicraTM transcatheter pacing system into the coronary sinus
}

\author{
Xueying Chen ${ }^{1}$, Jing-Feng Wang ${ }^{2}$, Yixiu Liang ${ }^{3}$, YANGANG SU${ }^{1}$, and JUN-BO GE ${ }^{3}$ \\ ${ }^{1}$ Zhongshan Hospital Fudan University \\ ${ }^{2}$ Zhongshan Hospital, Fudan University \\ ${ }^{3}$ Shanghai Institute of Cardiovascular Diseases, Zhongshan Hospital, Shanghai Medical \\ College of Fudan University
}

November 2, 2020

\begin{abstract}
Leadless pacemaker emerged as a new pacing strategy to avoid pocket and lead-related complications as compared with conventional pacemaker. Whereas, cardiac perforation remains a major safety concern during the implantation. Herein, we presented a rare case of cardiac perforation with pericardial tamponade caused by accidently placing the delivery catheter into the coronary sinus and she was successfully recovered after immediate pericardiocentesis and discharged without other adverse events.
\end{abstract}

\section{Introduction:}

Recently, leadless pacemaker emerged as a new pacing strategy to avoid pocket and lead-related complications as compared with conventional pacemaker ${ }^{1}$. However, though the incidence of major complications was demonstrated to be low to $1.51 \%$ in Micra transcatheter pacing system (TPS) (Medtronic, Minneapolis, $\mathrm{MN}, \mathrm{USA})^{2}$, cardiac perforation remains a major safety concern with the incidence of about $0.13 \%^{\sim} 1.3 \%$ in studies ${ }^{2,3}$. Herein, we presented a case of cardiac perforation with pericardial tamponade caused by accidently placing the delivery catheter into the coronary sinus.

\section{Case presentation:}

A 83 -year-old female (height, $148 \mathrm{~cm}$; weight, 41.5kg; body mass index,18.9 $\mathrm{kg}[?] \mathrm{m}^{-2}$ ) suffered from sinus arrest of 5 seconds with paroxysmal atrial flutter and was admitted to our hospital for leadless pacemaker implantation. The patient had percutaneous coronary intervention with 2 stents implantation 1 month before admission and dual anti-platelet therapy of aspirin (100mg/day) and clopidogrel (75mg/day) continued to the procedure day. During the procedure, the patient was received intravenous heparin $50 \mathrm{u} / \mathrm{kg}$ before Micra TPS was introduced into the right femoral vein. Then the delivery catheter was directed across the tricuspid valve but was accidently performed into the coronary sinus for several times. The device cup was placed at the beginning of the posterior branch of coronary sinus as confirmed by angiography (Figure 1 ). Simultaneously, pericardial effusion was detected at the left anterior oblique view (Figure 1B, 1C ). But the patient was asymptomatic and remained hemodynamically stable (blood pressure $134 / 76 \mathrm{mmHg}$ ). After adjusting the direction of the catheter, it was finally successfully performed into the right ventricle and Micra leadless pacemaker was deployed at the apex of right ventricle (Figure 2 ) with good pacing parameters ( $\mathrm{R}$ wave amplitude, $9 \mathrm{mV}$; threshold, $0.38 \mathrm{~V} / 0.24 \mathrm{~ms}$; impedance, $1000 \Omega$ ). At 2 hours post-procedure, the patient was found pericardial tamponade with blood pressure dropped to $75 / 58 \mathrm{mmHg}$. Medium amount of pericardial effusion mainly distribution around the posterior wall of left ventricle was confirmed by echocardiogram (Figure 3 ). The patient was emergently received pericardiocentesis and drainage of $270 \mathrm{ml}$ bloody fluid. The symptoms were immediately relieved with blood pressure rise to $130 / 80 \mathrm{mmHg}$. The pacing 
parameters remained stable. Dual anti-platelet therapy was stopped for 5 days. No evidence of distinct pericardial effusion was detected after the drainage tube removal. Aspirin (100mg/day) and rivaroxaban $(5 \mathrm{mg} /$ day $)$ were prescribed afterwards. The patient was discharged without pericardial effusion reconfirmed by echocardiogram.

\section{Discussion:}

Due to the different fixation way, cardiac perforation remains one of the severe complications of leadless pacemaker. It is recommended to implant it at the septum of the right ventricle to minimize the incidence of cardiac perforation ${ }^{2,4}$ though it is not easy to achieve in all patients, especially in small hearts or cor pendulum (drop hearts) cases. According to the literatures ${ }^{2,3}[2,3]$, the risk factors for cardiac perforation in leadless pacemaker included female, low body mass index, history of myocardial infarction and lung diseases. Therefore, each patient should be carefully estimated before implantation, especially in cases with these risk factors. Our patient was an old female with low body mass index on dual anti-platelet therapy, which increased the peri-procedure bleeding risk, although the study by Kiani $\mathrm{S}^{5}$ et al showed continuation of therapeutic anticoagulation during MICRA implantation was feasible and safe. It is challenging to implant the leadless pacemaker in small-size heart cases since the shape of the delivery catheter is fixed. In our patient, it is not easy to perform Micra TPS across the tricuspid valve and accidently place it into coronary sinus after multiple attempts. Left anterior oblique view and angiography were useful to distinguish Micra TPS locating at coronary sinus or right ventricle. Other than cardiac injury by the fixation apparatus after deployment of Micra, cardiac perforation resulting from the delivery catheter against the ventricular wall has also been illustrated. Togashi I ${ }^{6}$ et al reported a case of subclinical cardiac perforation caused by the edge of the device cup penetrating into the ventricular wall prior to the deployment of the leadless pacemaker. Another 91-year-old female case reported by Hai $\mathrm{JJ}^{4}$ et al developed cardiac perforation due to contrast injection against the RV anterior wall before verification of sheath location. The cause of pericardial effusion in the present case was probably the coronary vein injury by the edge of the device cup, since pericardial effusion was detected by angiography when the catheter was placed into coronary sinus (Figure 1 ). The pericardial effusion aggravated and pericardial tamponade occurred probably due to dual anti-platelet therapy together with anti-coagulation of heparin. In terms of short half-life period, bivalirudin might be more suitable than heparin for peri-implantation anti-coagulation in patients on dual anti-platelet therapy to reduce the bleeding risk.

\section{References}

1. El-Chami MF, Al-Samadi F, Clementy N, et al. Updated performance of the Micra transcatheter pacemaker in the real-world setting: A comparison to the investigational study and a transvenous historical control. Heart Rhythm. 2018;15(12):1800-1807.

2. Roberts PR, Clementy N, Al Samadi F, et al. A leadless pacemaker in the real-world setting: The Micra Transcatheter Pacing System Post-Approval Registry. Heart Rhythm. 2017;14(9):1375-1379.

3. Reynolds DW, Ritter P. A Leadless Intracardiac Transcatheter Pacing System. $N$ Engl J Med. 2016;374(26):2604-2605.

4. Hai JJ, Fang J, Tam CC, et al. Safety and feasibility of a midseptal implantation technique of a leadless pacemaker. Heart Rhythm.2019;16(6):896-902.

5. Kiani S, Black GB, Rao B, et al. Outcomes of Micra leadless pacemaker implantation with uninterrupted anticoagulation. J Cardiovasc Electrophysiol. 2019;30(8):1313-1318.

6. Togashi I, Sato T, Hoshida K, Soejima K. Subclinical cardiac perforation caused by a Micra leadless pacemaker. J Arrhythm.2018;34(3):326-328.

\section{Figure legends:}

Figure 1. Angiography showing Micra TPS placing into the coronary sinus: A. At poster-anterior view; Pericardial effusion (arrow) at left anterior oblique view for the first attempt (B) and the second attempt 
(C).

Figure 2. The images showing the location of Micra leadless pacemaker: A. After deployment at right anterior oblique view; B. CT scan after implantation indicating the leadless pacemaker locating at the apex of right ventricular

Figure 3. The echocardiogram showing pericardial effusion (red arrow) during diastolic phase mainly distribution around the posterior wall of left ventricle. 


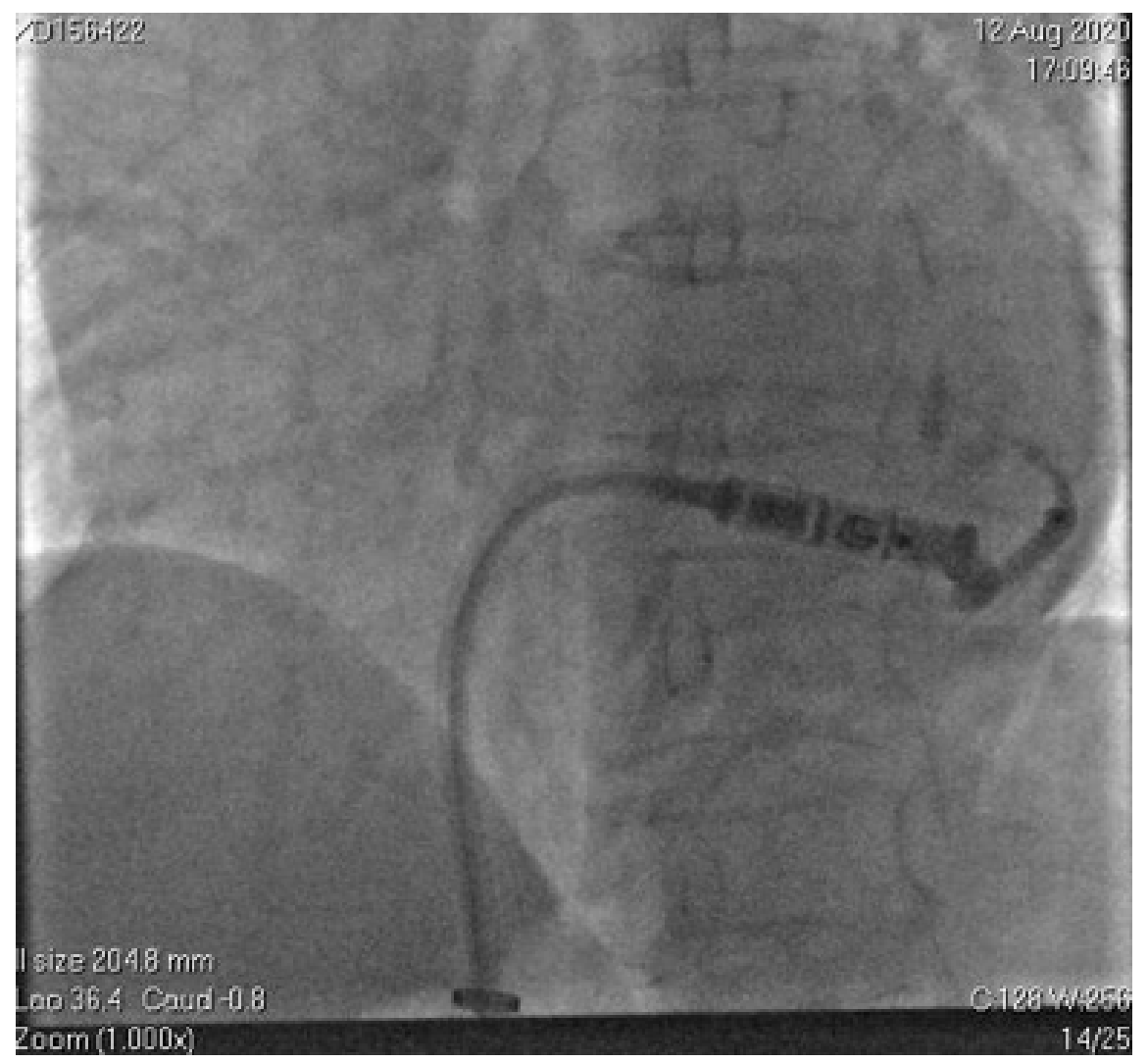



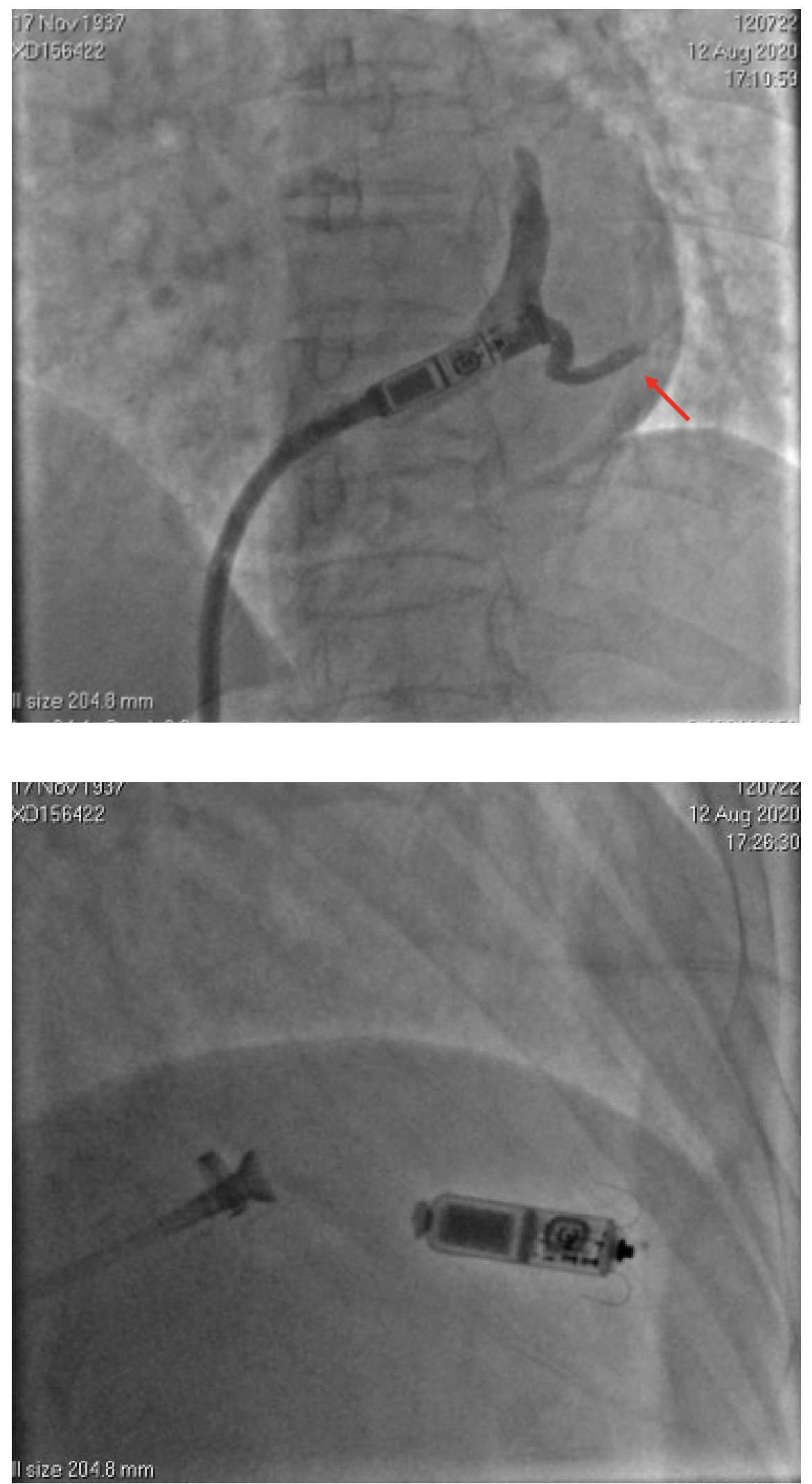

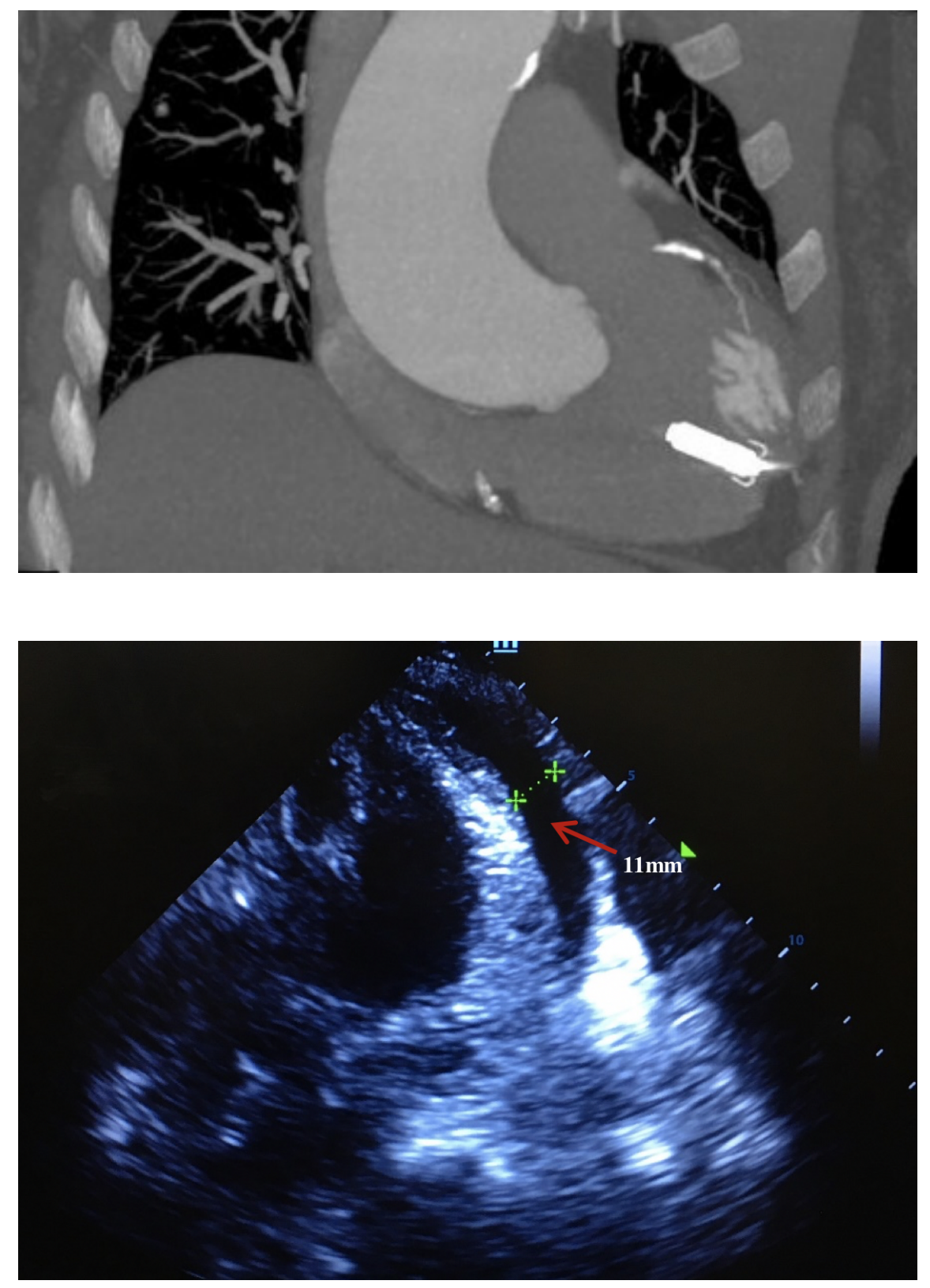